\title{
A HISTOPATHOLOGICAL STUDY OF ISCHEMIC AND COMPRESSIVE PARAPLEGIA IN DOGS
}

\author{
Šulla, I. ${ }^{1}$, Balik, V. ${ }^{2}$, Maženský, D. ${ }^{1}$, Danielisová, V. ${ }^{3}$ \\ ${ }^{1}$ Department of Anatomy, Histology and Physiology \\ University of Veterinary Medicine and Pharmacy, Komenského 73, 04181 Košice \\ Slovakia \\ ${ }^{2}$ Department of Neurosurgery, Faculty Hospital, I. P. Pavlova 13, 77900 Olomouc \\ Czechia \\ ${ }^{3}$ Institute of Neurobiology, Slovak Academy of Sciences \\ Šoltésovej 4-6, 04001 Košice \\ Slovakia
}

igor.sulla@uvlf.sk

\section{ABSTRACT}

It is well known that neuronal death, clinically manifested as paresis or plegia, is the end result of many pathological events affecting the central nervous system. However, several aspects of pathophysiological mechanisms involved in the development of tetra- or paraplegia caused by spinal cord traumatic or ischemic damage are only insufficiently understood and their histopathological manifestations remain poorly documented. That is why the authors decided to report on light-microscopic changes observed in $30 \mu \mathrm{m}$ thick spinal cord sections cut from L3-S1 segments processed by the Nauta staining method in a group of 6 dogs with ischemic paraplegia induced by $30 \mathrm{~min}$ of a high thoracic aorta occlusion, and in a different group of 6 dogs with traumatic paraplegia induced by $5 \mathrm{~min}$ spinal cord compression with $200 \mathrm{~g}$ metallic rod. Both experimental groups (ischemic and compression) of spinal cord injuries (SCI) comprised the same number of mongrel dogs of both sexes, weighing $18-25 \mathrm{~kg}$. In addition, each of the experimen- tal groups had 3 normal dogs that served as controls. All experimental procedures were accomplished under general anaesthesia induced by pentobarbital and maintained by a mixture of halothane and oxygen. Following the 72 hour survival period, all 18 animals were euthanized by transcardial perfusion with $3,000 \mathrm{ml}$ of saline and fixed by $3,000 \mathrm{ml}$ of $10 \%$ neutral formaldehyde during deep pentobarbital anaesthesia. The histopathological manifestation of neural tissue damage caused by ischemia or compression was similar. The light-microscopic images in both groups were characterised by argyrophilia and the swelling of grey matter neurons. However, in the dogs with traumatic SCIs, the changes only reached about $750 \mu \mathrm{m}$ cranially and caudally from the necrotic epicentre. These findings indicated that the events taking part in secondary spinal cord injury mechanisms are similar in both, ischemic as well as in traumatic SCI.

Key words: compression; histopathology; ischemiareperfusion; spinal cord injury; 


\section{INTRODUCTION}

Spinal cord injuries, whether caused by external traumatic forces or ischemic events belong to the most serious healthcare problems in both, human and veterinary health care practices $[1,4,6,8,18]$. Due to their devastating effects on motor, sensitive and autonomic neural functions, SCI have attracted the interest of the people (from ancient physicians and philosophers, to modern scientists, veterinarians, as well as laymen and the public) for a long time $[2,13$, $14,15]$. Despite an increasing amount of information accumulated during the last several decades, many aspects of the pathophysiological mechanisms involved in the development of tetra- or paraplegia have only been partially elucidated and their neurohistological manifestations remain incompletely documented $[7,14,18,21]$. This situation inspired the authors to study light-microscopic changes characteristic for spinal cord damage induced by two different types of insults to the spinal cord, i. e. ischemia-reperfusion and compression injuries, in two different canine experimental models.

\section{MATERIALS AND METHODS}

The experimental protocols were prepared with respects to the instructions of the Animal Protection Act of the Slovak Republic No. 15/1995 [5] and approved by the Ethical Commission of the Institute of Neurobiology, Slovak Academy of Sciences in Košice, Slovakia.

Eighteen healthy adult mongrel dogs of both sexes, free of heart worm disease, weighing $18-25 \mathrm{~kg}$, divided into four groups, were used in this study:

1. Non-ischaemic sham controls $(n=3)$. Animals underwent a left-sided thoracotomy only.

2. Non-compressive sham controls $(n=3)$. The standard L4-L5 laminectomy was performed.

3. Ischemic injuries of the spinal cord $(n=6)$. The descending aorta just distal to the origin of the left subclavian artery was occluded by a tourniquet for $30 \mathrm{~min}$.

4. Compression SCI $(n=6)$. The spinal cord was traumatised by a metallic rod ended with a small knob weighing $200 \mathrm{~g}$ lowered onto the exposed spinal dural sac containing the spinal cord through the L4L5 laminectomy for $5 \mathrm{~min}$.
The experimental procedures were carried out under general anaesthesia induced by Pentobarbital (pentobarbitalum natricum) administered intravenously in a $30 \mathrm{mg} \cdot \mathrm{kg}^{-1} \cdot 10^{-1}$ dose. Then the dogs were intubated with an endotracheal cannula (diameter 8-12 $\mathrm{mm}$ ) and placed on a volume-cycled ventilator. The anaesthesia was further maintained by a mixture of oxygen and Halothane. Also, the continuous direct monitoring of the arterial blood pressure in the radial artery, EKG, and arterial blood gases were carried out. The rate of ventilation was adjusted to maintain arterial $\mathrm{pO}_{2}$ between $80-100 \mathrm{~mm} \mathrm{Hg}$ and $\mathrm{pCO}_{2}$ at about $38 \mathrm{~mm} \mathrm{Hg}$, i. e., normal canine levels of $\mathrm{pO}_{2}$ and $\mathrm{pCO}_{2}$ [19]. The arterial blood pressure (which increased in every dog after a high thoracic aorta occlusion) was kept at pre-surgical levels by a continuous infusion of nitroprusside in saline.

The operations were performed under sterile conditions and basic surgical principles were strictly observed. Antibiotics (1,500,000 u. i. of benzylpenicillinum procainum and $1.5 \mathrm{~g}$ of streptomycin sulphate) were administered intramuscularly during the surgery to every dog. After completing the surgical procedures, the dogs were placed into separate disinfected compartments, covered with warm blankets, and followed until they completely awakened from the narcosis. Then, they were offered drinking water ad libitum and food granules. Suppression of postoperative pain was ensured by the intramuscular administration of a strong analgesic/opioid (Tramal, i. e. tramadoli hydrochloridum $50 \mathrm{mg}$ ) in 6 hour intervals for 3 days.

After a 72 hour survival period, all dogs (experimental as well as controls) were deeply anaesthetized by pentobarbital ( $50 \mathrm{mg} \cdot \mathrm{kg}^{-1} \cdot 10^{-1}$ of body weight i.v.), then transcardially perfused with $3,000 \mathrm{ml}$ of saline and fixed by the same volume of $10 \%$ neutral formaldehyde. Spinal cord specimens comprised of L3-S1 segments were removed, 24 hours post-fixation, cut into $30 \mu \mathrm{m}$ thick sections and processed by silver compounds according to a protocol of Nauta staining methods for light-microscopic observations [12].

\section{RESULTS}

All 18 dogs survived the experimental procedures without surgical complications. All six control dogs revealed no neurological deficit. On the other hand, all of the experimental animals awakened from anaesthesia with fully 
developed paraplegia; their clinical picture was characterized by a complete palsy of their pelvic limbs. Parts of the spinal cord located in the epicentre of the compression injury in members of this experimental group (6 dogs), were crushed; the appearance of the neural tissue resembled an engorged haemorrhagic pulp. The spinal cord in the vicinity of the trauma was oedematous and haemorrhagic, but macroscopic changes did not exceed $14 \mathrm{~mm}$ cranially and caudally from the epicentre. The L3-S1 segments of the spinal cords removed from the vertebral canals of members of the ischemic group ( $6 \mathrm{dogs}$ ) were pale and oedematous, but not haemorrhagic. The macroscopic changes were not restricted to the epicentre and 1-2 adjacent segments only, but affected the whole specimen.

\section{Light-microscopic observations}

No visible histopathological changes developed in the specimens prepared from the lumbosacral spinal cord segments of the sham controls. The identity of neurons in semi-thick sections $(30 \mu \mathrm{m})$ of the L3-S1 spinal cord segments of sham controls processed according to the Nauta staining method was hardly distinguishable. The perikarya were only minimally impregnated and their dendritic ramifications were completely Nauta-negative. The cytoplasm of middle-sized and large neural cells (especially the large motoneurons in Rexed's lamina IX) appeared as pale shadows, almost identical with the background [17]. The localisation of neural cell somata was facilitated by large light nuclei and darkly stained nucleoli (Fig. 1).

The histopathological changes of the spinal cord tissue damaged by $30 \mathrm{~min}$ of ischemia and 72 hour reperfusion on the one hand, and $5 \mathrm{~min}$ compression by $200 \mathrm{~g}$ metallic rod on the other hand, were similar with only insignificant differences. The histopathological images were characterised by argyrophilia of the neural cells, as well as swelling and boutonic enlargement of presynaptic parts of their axons encircled by clear halos (Figs. 2-4). The majority of boutons was localised in the central part of spinal cord grey matter, i.e. Rexed's laminae V-VII [17]. The above changes were diffusely spread throughout the spinal cord grey matter in both experimental groups, but in the dogs with compressive paraplegia, they did not exceed $750 \mu \mathrm{m}$ cranially and caudally from the necrotic epicentre.

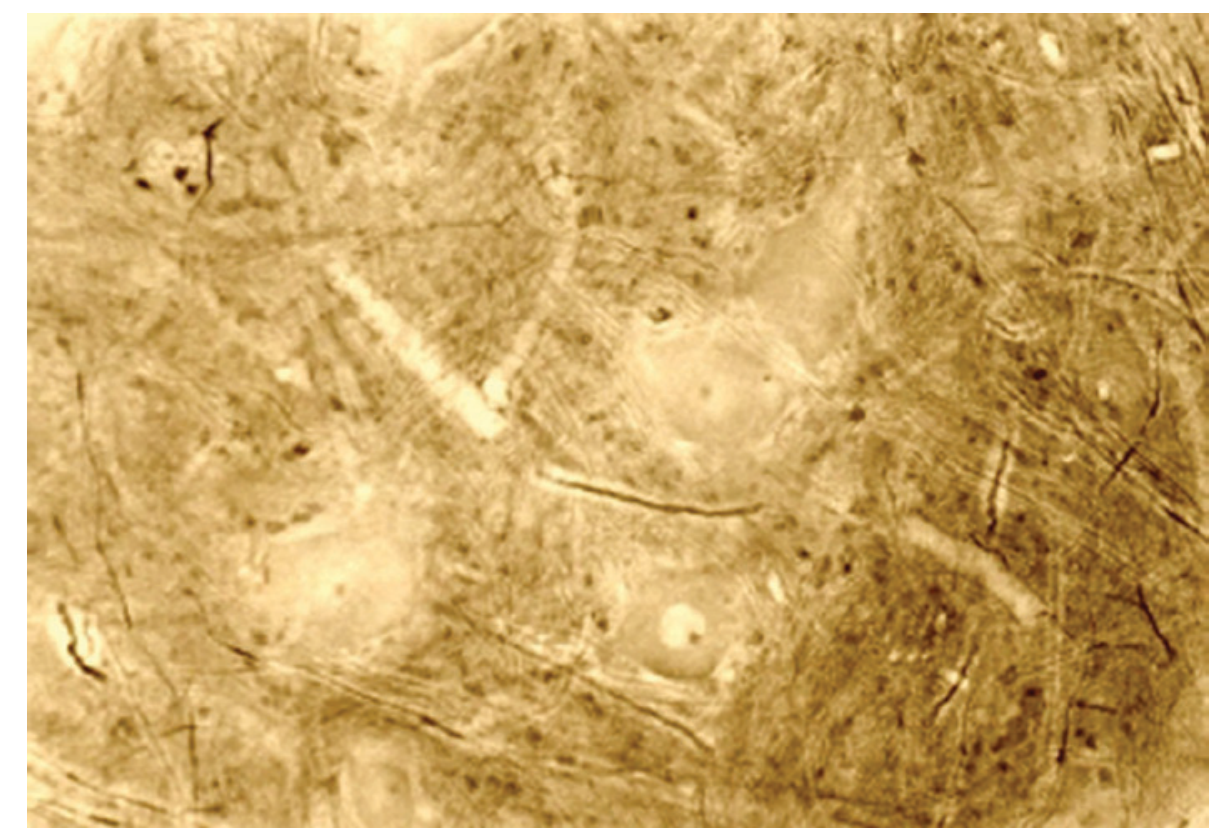

Fig. 1. Microphotograph of a specimen from L5 spinal cord segment of a control dog. Large motoneurons with darker nucleoli are Nauta-negative and appear as pale shadows, almost identical with surrounding neuropil. Magn. $\times 180$ 


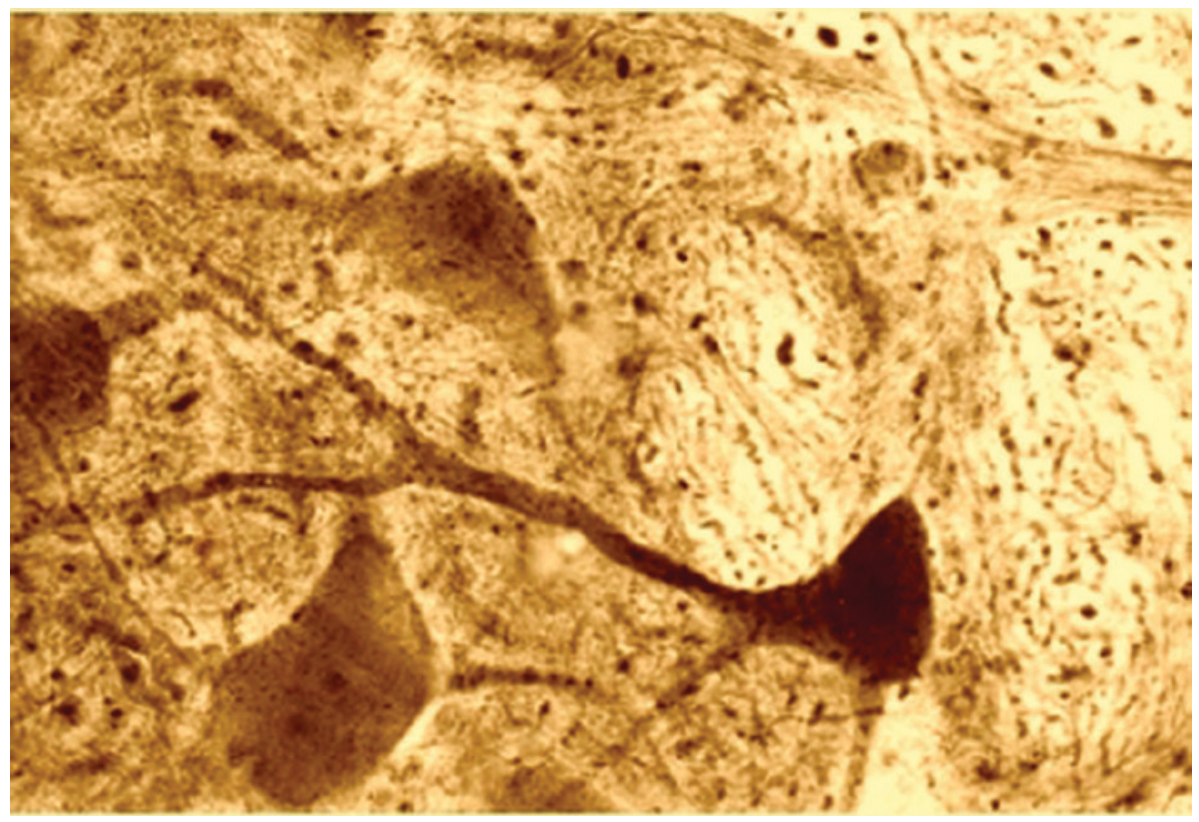

Fig. 2. Microphotograph of a specimen from L5 spinal cord segment of a dog after 30 min ischemia and 72 hour survival. Oedema and enhanced argyrophilia of interneurons in zona intermedia. Magn, $\times 320$

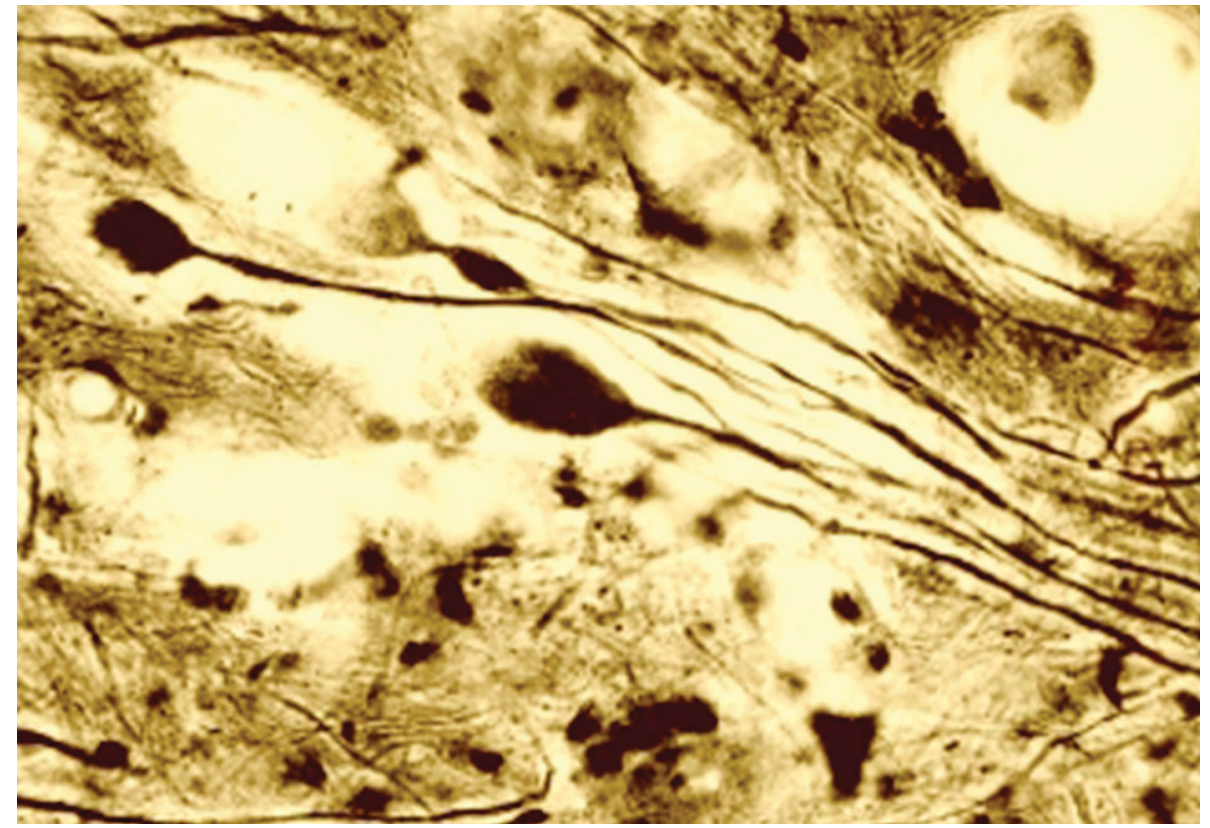

Fig. 3. Microphotograph of a specimen from L5 spinal cord segment of an animal after $30 \mathrm{~min}$ ischemia and 72 hour survival. Enlarged terminal parts of axons (boutons) in the 6th Rexed's lamina of the dorsal horn. Magn. $\times 150$ 


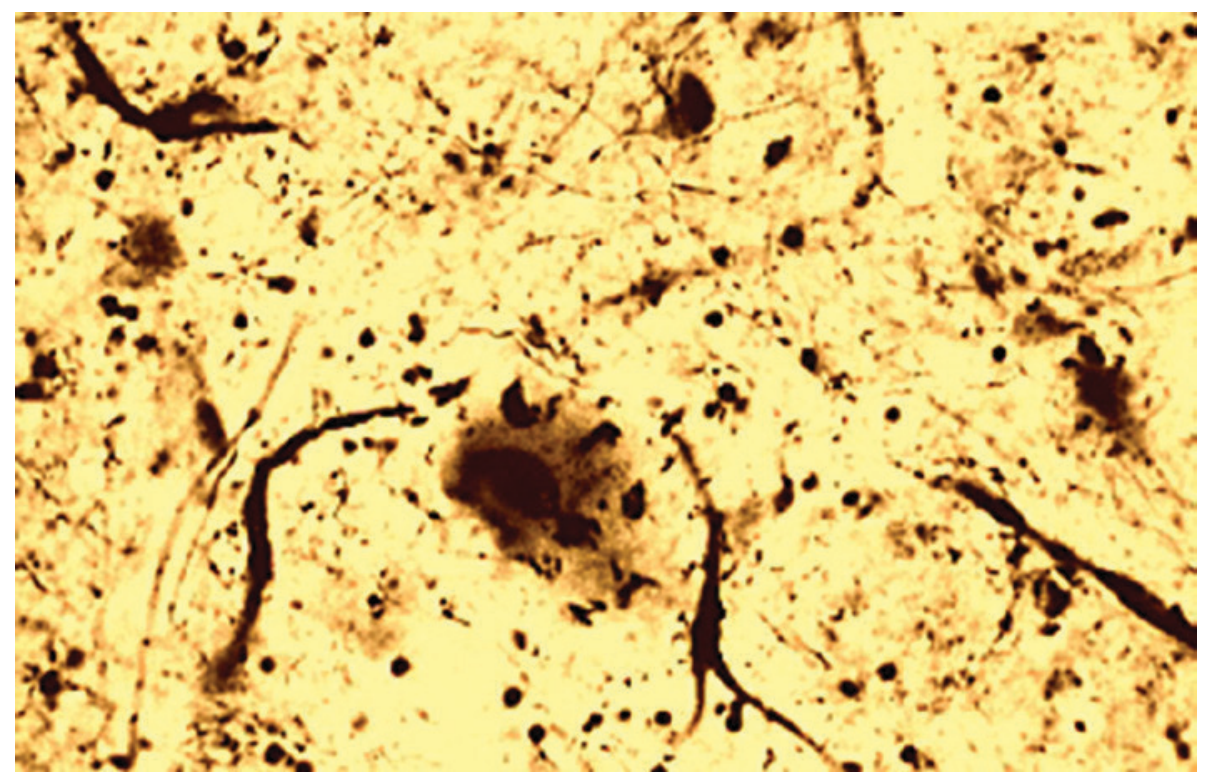

Fig. 4. Microphotograph of a specimen from the L3 spinal cord segment of an animal following the 5 min of compression trauma and 72 hour survival. Neuropil is Nauta-positive, motoneurons in anterior horns are oedematous, and within their cytoplasm are argyrophilic accumulations of irregular shape and density. Magn. $\times 120$

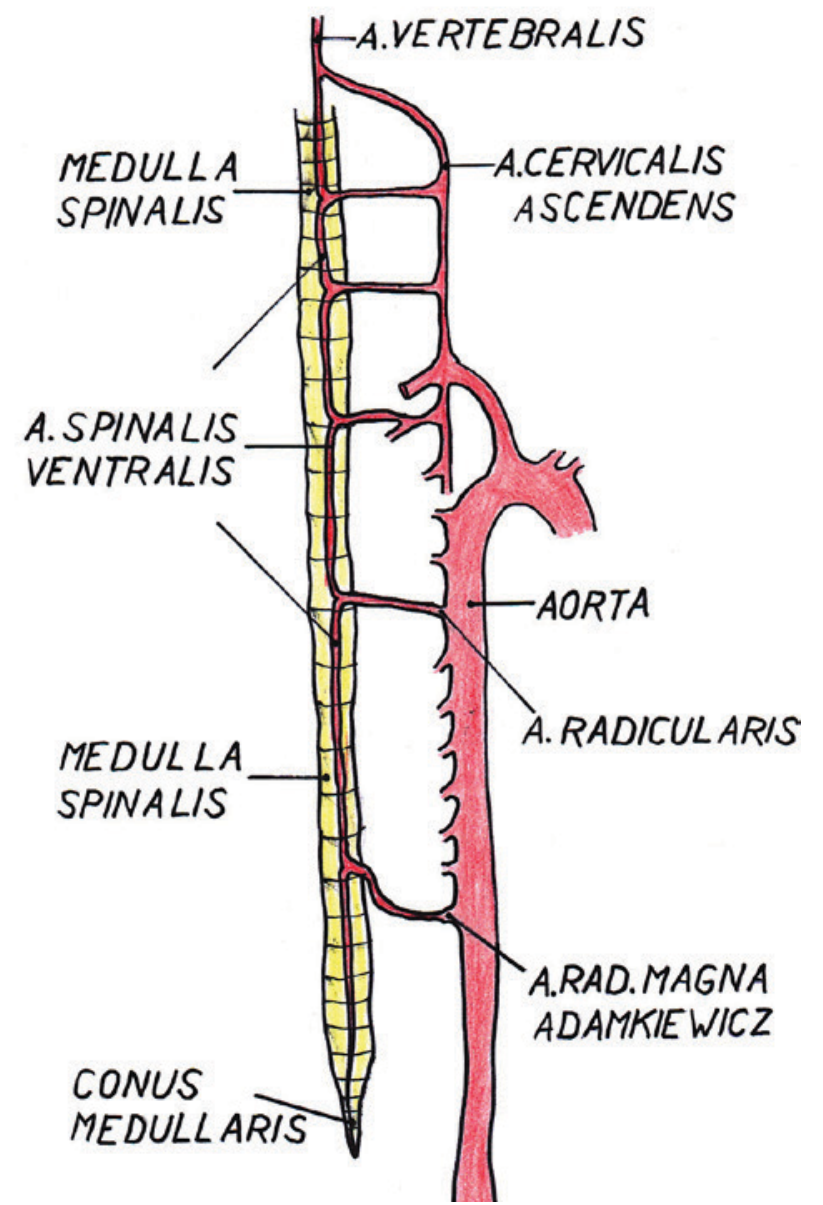

Fig. 5. Schematic drawing of arteries supplying the spinal cord modified after La z or th es et al. [9] 


\section{DISCUSSION}

The terms tetraplegia and paraplegia refer in mammals to the permanent deficit of motor functions of the trunk, all four extremities and tail (tetraplegia) or pelvic limbs and tail muscles palsy (paraplegia) accompanied by a loss of: voluntary control over the urinary bladder and anal sphincters' functions, sexual incompetence as well as a complete anaesthesia of the affected body area $[6,7,8]$. A very similar neurological deficit is characteristic for spinal cord lesions in humans $[1,18,21]$. Trauma to the vertebral column and spinal cord plays the main role in the development of the clinical syndrome; less frequently it is caused by an interruption of spinal cord blood flow $[8,11,13,18]$.

The human spinal cord is supplied by 1 ventral and 2 dorsal spinal arteries, which extend longitudinally in a variable fashion $[9,22]$. Cranially these arteries originate from the vertebral arteries and anastomose at the level of the conus medullaris. At many levels they receive blood supplies from the radicular arteries, which enter the vertebral canal along the nerve roots. The anterior spinal artery gives rise to the central arteries, entering the spinal cord to supply the ventral horns and ventral parts of the lateral columns. Each radicular artery supplies a separate functional region of the spine, particularly via the ventral spinal artery. The first region extends from $\mathrm{C} 1$ to the Th3 segment and is supplied at the C3 level from the vertebral arteries. The second region extends from Th3 until the Th7 and receives a branch from the intercostal artery (originating directly from the aorta) at the Th7 level. The third region, supplied by only one branch, arteria radicularis magna (Adamkiewicz artery) originating from an intercostal or lumbar artery between Th9, L1, L2, extends from Th8 to the conus medullaris (Fig. 5). This arrangement of the spinal cord blood supply explains why even a temporary cross-clamping of the thoracic aorta can cause necrosis of the medullary tissue and lead to the development of ischemia-reperfusion paraplegia, characterized by bilateral motor deficit with complete sensory deficit due to the so called transverse medullary infarct [22].

The temporary cross-clamping is a condition sine qua non in the aortic or principal vessels operations $[1,11,16]$. However, awakened from anaesthesia after an uneventful, technically straightforward procedure with an irreversible paraplegia, means a catastrophe for a patient and a nightmare for the vascular surgeon. The risk of this complication is about $10 \%$ in elective thoracoabdominal aortic recon- struction and significantly increases in patients struck with a rupture of an atherosclerotic aneurysm of the vessel [1, 11]. The reason is that haemorrhagic shock causing a rapid decrease of blood pressure which, in combination with preexisting latent insufficiencies of the spinal cord vessels and their supplying arteries, is an extremely malignant combination $[8,13,16]$.

The experimental model of spinal cord ischemia-reperfusion injury used in our study was developed with an aim to imitate the situation in humans as much as possible. Originally we considered the utilisation of small laboratory animals in our experiments [10]. We had both, rabbits, as well as rats at our disposal. However, interventions in the thoracic cavity of rabbits or rats require a special apparatus for maintaining artificial ventilation and an endotracheal intubation which is so technically difficult, that a cannula often can be introduced into trachea through a tracheotomy only. Moreover, in rabbits another problem appears; the spinal cord lesion leads to a complete urinary retention, which causes an early death of the animal [10]. On the other hand, administration of general anaesthesia to larger animals is simpler; the surgical situation in a thoracic cavity resembles the situation in humans, and as a result are more reliable. That is why minipigs, dogs or cats are used more and more frequently in experimental studies nowadays [16, 20]. Having at our disposal the necessary equipment for intrathoracic and intraspinal procedures in dogs, as well as sufficient experience with operations on them, we decided to use these animals again [19].

The pathophysiological consequences of spinal cord damage are explained by the theory of primary and secondary injury mechanisms. Primary SCI result from the direct mechanical insults to neural tissues and spinal cord (especially grey matter) vascular network $[15,21]$. Four basic types of primary SCI and their combinations can occur. They include: the laceration of the spinal cord (caused by shearing and traction), compression of the cord (by an intraspinal haematoma, herniated intervertebral disc, dislocated vertebra or its fragments in a case of vertebral fracture or luxation), concussion of the spine (e.g. when the victim was hit by an automobile, gunshot or falling from a height), and lack of blood supply, i. e. ischaemia $[3,4,6$, $7,11]$. The secondary mechanism in SCI develops as an expansion of the primary damage [14]. Regardless of the type of insult, which started the cascade of events leading to disintegration of spinal cord tissue (neurons and sup- 
portive glial cells), and destruction of connections between brain - spinal cord and spinal cord - spinal cord neurons, the secondary mechanism in SCI develop as an expansion of primary damage $[13,14,15]$. The vast majority of investigators comply with the theory of a multi-step process, including: persistent depolarization of cell membranes, energy depletion from repolarization, deregulation of intracellular calcium, formation of reactive oxygen species, lipid peroxidation, loss of cellular ionic gradients, oedema formation, nuclear DNA fragmentation, rupture of cell membranes and inflammatory reaction in both, traumatic and ischemic SCIs $[3,8,14,18,21]$. An important finding of our study was the ability of the Nauta staining method to visualize and localize damaged spinal cord neurons when their somata and dendrites became argyrophilic due to an ischemia-reperfusion or a compression injury. The unusual type of synaptic degeneration, characterized by a massive occurrence of enlarged boutons encircled by clear halos, could be a light-microscopic manifestation of ionic shifts and membrane depolarization processes, which are considered an integral part of a cascade leading to neuronal death in SCI. The density and laminar distribution of boutons correlated with the distribution of small argyrophilic neurons localized in the deep dorsal horn layers. The results of our experiments demonstrated the high vulnerability of these spinal cord grey matter cells. With regard to the fact, that many small neurons and interneurons form synapses with axons of descending supraspinal tracts, the damage induced by ischemia-reperfusion and compression SCI very probably play an important role in the gradual transformation of post-ischemic or compressive paraplegia from a flaccid paralysis to a spastic palsy [14, 21, 22].

More than a decade ago, spinal cord traumatic or ischemia-reperfusion lesions resulted in confinement of the patients to wheelchairs and struggles with different medical complications for the rest of their life $[1,6,7,8,9,13$, 21]. The treatment armamentarium was limited and the provision of care for individuals with SCI was met with frustration in the majority of cases. Progress in neuroscience research has made the idea, that SCI will eventually be treatable, more plausible $[3,14,16,18]$.

The authors believe that the histopathological changes observed in the spinal cord specimens of dogs of both experimental groups, expressed the consequences of compromised spinal microcirculation. It means that ischemia plays an important role in the pathophysiology of both, traumatic and ischemia-reperfusion SCI. Therapeutic measures need to take this observation into consideration.

\section{CONCLUSIONS}

The comparison of neurohistopathological changes observed in specimens prepared from L3-S1 spinal cord segments of dogs with ischemic and compressive paraplegia stained by the Nauta method indicated that metabolic events leading to cell death in these different experimental models are similar and certain ischemic components also participate in development of traumatic spinal cord lesions.

\section{REFERENCES}

1. Acher, C., Wynn, M., 2012: Paraplegia after thoracoabdominal aortic surgery: not just assisted circulation, hypothermic arrest, clamp and sew, or TEVAR. Ann. Cardiothorac. Surg., $1,365-372$.

2. Adams, F., 1849: The Genuine Works of Hippocrates. Translated from Greek. Section II. Hippocratic Treatises. S. 24-132. London, Sydenham Society.

3. Ahuja, C.S., Fehlings, M., 2016: Concise review: Bridging the gap: Novel neuroregenerative and neuroprotective strategies in spinal cord injury. Stem Cell Transl. Med., 5, 914-924.

4. Albin, M.S., White, R.J., 2000: Therapeutic window after spinal cord trauma is longer than after spinal cord ischemia. Anaesthesiology, 92, 281-282.

5. Animal Protection Acta of the Slovak Republic No. 15/1995 (In Slovak), part 39, 1250-1255.

6. Bruce, C. W., Brisson, B. A., Gyselinck, K., 2008: Spinal fracture and luxation in dogs and cats: a retrospective evaluation of 95 cases. Vet. Comp. Orthop. Traumatol., 21, 280-284.

7. Henke, D., Vandervelde, M., Doherr, M. G., Stickli, M., Forterre, F., 2013: Correlations between severity of clinical signs and histopathological changes in 60 dogs with spinal cord injury associated with acute thoracolumbar intervertebral disc disease. Vet. J., 198, 70-75.

8. Ishikawa, T., Suzuki, H., Ishikawa, K., Yasuda, S., Matsui, T., Yamamoto, M. et al., 2014: Spinal cord ischemia/injury. Curr. Pharm. Des., 20, 5738-5743.

9. Lazorthes, G., Gouazé, A., Zadeh, J.G., Lantini, J., Lazorthes, J., Bardin, P., 1971: Arterial vascularisation of the spinal cord. J. Neurosurg., 35, 253-262. 
10. Maženský, D., Flešárová, S., 2016: Importance of the arterial blood supply to the rabbit and guinea pig spinal cord in experimental ischemia. In Schaller, B. (Ed.): Ischemic Stroke Updates. Zagreb, Croatia, Tech., S. 59-86.

11. Martín, C., Forteza, A., Navarro, M., Cortina, J., 2007: Acute spinal cord ischemia following surgery for DeBakey type-1 dissection of the ascending aorta. Rev. Esp. Cardiol., 60, 1102-1007.

12. Nauta, W.J.H., 1957: Silver impregnation of degenerating axons. In Windle, W.P. (Eds.): New Research Techniques of Neuroanatomy. Springfield, Ill., Charles C. Thomas Publ., S. 17-26.

13. Nedeltchev, K., Loher, T. J., Stepper, F., Arnold, M., Schroth, G., Mattie, H.P., Sturzenegger, M., 2004: Long-term outcome of acute spinal cord ischemia syndrome. Stroke, 35, $560-565$.

14. Oyinbo, C.A., 2011: Secondary injury mechanisms in traumatic spinal cord injury: a nugget of this multiply cascade. Acta Neurobiol. Exp., 71, 281-299.

15. Popa, C., Popa, F., Grigorean, V. T., Onose, G., Sandu, A. N., Popescu, M., et al., 2010: Vascular dysfunction following spinal cord injury. J. Med. Life, 3, 275-285.
16. Radoňák, J., Čížková, D., Lukáčová, N., Kluchová, D., Ostró, A., Gálik, J., 2009: Preconditioning as a possible protective mechanism in the spinal cord ischemia. Acta Vet. Brno, 78, 307-311.

17. Rexed, B., 1957: A cytoarchitectonic atlas of the spinal cord in the cat. J. Comp. Neurol., 100, 297-379.

18. Silva, N. A., Susa, N., Reis, R. L., Salgado, A. J., 2014: From basics to clinical: A comprehensive review on spinal cord injury. Progr. Neurobiol, 114, 25-57.

19. Šulla, I., Vanický, I., 2002: Laminectomy in canine experiments (In Slovak). Acta Spondylologica, 1, 109-112.

20. Šulla, I., Bačiak, I., Juránek, I., Cicholesová, T., Boldižár, M., Balik, V., Lukáčová, N., 2014: Assessment of motor recovery and MRI correlates in a porcine spinal cord injury model. Acta Vet. Brno, 83, 393-397.

21. Tator, C.H., Fehlings, M. G., 1991: Review of the secondary injury theory of acute spinal cord trauma with emphasis on vascular mechanisms. J. Neurosurg., 75, 15-26.

22. Zülch, K. J., Kurth-Schimacher, R., 1970: The pathogenesis of intermittent spinovascular insufficiency ("spinal claudication of Dejerine") and other vascular syndromes of the spinal cord. Vasc. Surg., 4, 113-136.

Received January 11, 2017

Accepted April 18, 2017 